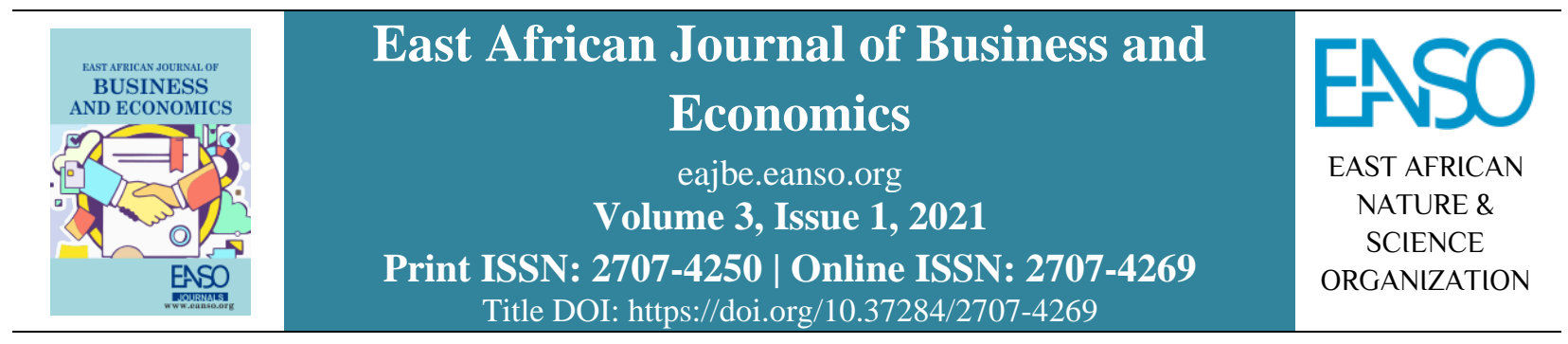

Original Article

\title{
The Determinants of Household Spending on Alcohol and Tobacco in Tanzania.
}

\author{
Stephen Richard Mwaisakila ${ }^{* *} \&$ Dr. Vid Adrison, PhD ${ }^{2}$ \\ ${ }^{1}$ Universitas Indonesia, Salemba Campus, Jakarta, 10430, Indonesia . \\ ${ }^{2}$ Universitas Indonesia, Depok Campus, West Java, 16424, Indonesia. \\ * ORCID : https://orcid.org/0000-0001-6298-8083; Correspondance email : mwaisakila21@ gmail.com.
}

Article DOI: https://doi.org/10.37284/eajbe.3.1.419

\section{Date Published: ABSTRACT}

23 September 2021 The increasing effects caused by alcohol and tobacco calls for necessary interventions. This is not only in developed countries, but also in developing

Keywords: countries which seems to be affected more by this problem. Studies show that $80 \%$ of smokers worldwide are living in developing countries where tobacco

Alcohol, related diseases have become major factors contributing toward high morbidity. Moreover, excessive alcohol consumption has been among global public health Determinants, problems, where it accounts for about $6 \%$ of mortality and $5 \%$ of disability in Household, the world. In addition, the prevalence of Alcohol Use Disorders (AUD) was reported at $4 \%$ globally while in Africa it was 3\%. Tanzania being the

Spending, developing country and located in Africa is also at risk to be affected more if the Tobacco. necessary measures to control this unhealthy behaviour are not taken. However, due to limited information about the structure and behaviour of people in spending and consumption of these addictive products in Tanzania, it will be difficult to prepare relevant measures to curb those problems. Therefore, by using Tobit regression model, this study examined the influence of socioeconomic characteristics on alcohol and tobacco spending. This study found factors such income, culture, and change in preference over time to have a significant relationship with spending on alcohol and tobacco. This study creates a base for alcohol and tobacco spending behaviour in Tanzania. After determining spending behaviour, further studies need to be conducted to examine consumption behaviour and its effects to prepare relevant measures to control the risks of alcohol and tobacco in Tanzania.

\section{APA CITATION}

Mwaisakila, S.R., \& Adrison, V. (2021). The Determinants of Household Spending on Alcohol and Tobacco in Tanzania. East African Journal of Business and Economics, 3(1), 173-186. https://doi.org/10.37284/eajbe.3.1.419

\section{CHICAGO CITATION}

Mwaisakila, Stephen Richard, \& Vid Adrison. 2021. "The Determinants of Household Spending on Alcohol and Tobacco in Tanzania”. East African Journal of Business and Economics 3 (1), 173-186. https://doi.org/10.37284/eajbe.3.1.419. 


\section{HARVARD CITATION}

Mwaisakila, S. R., \& Adrison, V. (2021) "The Determinants of Household Spending on Alcohol and Tobacco in Tanzania", East African Journal of Business and Economics, 3(1), pp. 173-186. doi: 10.37284/eajbe.3.1.419.

\section{IEEE CITATION}

S. R. Mwaisakila, \& V. Adrison, "The Determinants of Household Spending on Alcohol and Tobacco in Tanzania", EAJBE, vol. 3, no. 1, pp. 173-186, Sep. 2021.

\section{MLA CITATION}

Mwaisakila, Stephen Richard, \& Vid Adrison. "The Determinants of Household Spending on Alcohol and Tobacco in Tanzania". East African Journal of Business and Economics, Vol. 3, no. 1, Sep. 2021, pp. 173-186, doi:10.37284/eajbe.3.1.419

\section{INTRODUCTION}

At least $80 \%$ of smokers in the world reside in developing countries where tobacco-related illnesses have become one of the major factors contributing towards high morbidity (John, 2006). According to World Bank in 2019, they estimated that up to 2030 tobacco will account for the death of about 8 million people per year worldwide if the current trend persists while $80 \%$ of those premature death coming from developing countries (World Bank, 2019). Moreover, John (2005) argued that consumption of tobacco has the potential to drive the poor to the trap and a difficult circle of poverty and diseases. The reason behind is the existence of high morbidity and mortality associated with tobacco which its perseverance is higher among countries which are economically poor. In addition, the high morbidity and mortality caused by tobaccorelated diseases make it difficult for developing countries to achieve the Millennium Development Goals (MDGs) (John, 2005).

Furthermore, among global public health problems, excessive alcohol consumption is among them where it accounts for about $6 \%$ of mortality and 5\% of disability in the world (WHO, 2014). According to $\mathrm{WHO}$, about $53 \%$ of youth aging from 15 years and above are estimated to have occasionally consumed alcohol in their lifetime where, 39\% among them consumed even in the last year. The global estimates are not far from Africans' situation where in their lifetime, $43 \%$ of youth 15 years and above have occasionally consumed alcohol while among them, 30\% have even consumed it in the last year (Francis et al., 2015).
Moreover, the prevalence of Alcohol Use Disorders (AUD) reported at $4 \%$ globally while in Africa is $3 \%$ with high prevalence among men (WHO, 2014). Basically, AUD are serious problems in human health since they relate to severe and long-term medical complications. The effects of AUD may also interfere with the treatment of chronic diseases such as diabetes and HIV/AIDS if there is poor adherence of treatment (Ahmed et al., 2006). Risk situations and problems such as unintentional injuries, domestic violence, unemployment and decrease in work productivity are also associated with Alcohol use and AUD (Francis et al., 2015).

Not only developing countries, but also developed countries are also suffering from excessive alcohol consumption problems. Evidence from developed countries revealed that the behaviour of overconsuming alcohol often begins at early ages (Swahn et al., 2010). The evidence can be seen from the 2012 WHO report where $46 \%$ of the world's adolescents aged between 15 and 19 years reported having occasionally consuming alcohol, and 34\% consumed it in the last year while in Africa, the prevalence was $41 \%$ for ever used alcohol and $29 \%$ for those consumed even in the last year. In addition, the global prevalence of heavy episodic drinking in young age was $8 \%$ while in Africa was $6 \%$ and being higher among young age than adults (WHO, 2014).

The efforts of the WHO to reduce harmful effects resulting from the use of alcohol, tobacco and illegal use of drugs has started since 1992. Since that time WHO has advocated different approaches in order to reduce the adverse effects of alcohol, tobacco, 
and drug use. The efforts resulted in positive outcomes in some few countries like Italy where a negative trend in demands of alcohol and tobacco have been experienced since 1985 (Pierani \& Tiezzi, 2009). However, further efforts are needed to alleviate the public health care costs implied by the adverse consequences and negative externalities caused by alcohol, tobacco, and drug consumptions. These negative externalities include the effects on crime, or injuries caused by motor vehicle accidents and on labour market performance which costs the economy a lot.

To reduce the adverse impacts caused by tobacco and alcohol consumptions, policy makers need a clear picture about how this problem persists. It is necessary to understand the patterns on how individuals and households allocate their expenditure on alcohol and tobacco. Moreover, to prepare relevant policy to combat the negative effects caused by tobacco and alcohol consumption, it is necessary to understand the problems clearly as well as factors which can influence the decision.

\section{EMPIRICAL REVIEW}

Previous study found factors related to sociodemographic, economic, environmental, personal, and indicators of healthy life to be among the significant factors that influence tobacco consumption in the group of people over 15 years. Moreover, factors such as age, gender, education, marital status, fruit consumption, exposure to tobacco smoke at home, and frequent alcohol consumption are the most influential variables affecting tobacco consumption (Alkan \& Abar, 2020).

Moreover, previous literature revealed the existence of a strong habit which affects both alcohol and tobacco use while demand for both goods reflect a forward-looking behaviour (Pierani \& Tiezzi, 2009). In addition, the study revealed that alcohol and tobacco are complementary goods after finding the cross-price elasticity of both products to be negative. The argument behind these results is that a policy measure which can be effective in reducing the demand of one among two products, will eventually produce additional results on reducing consumption of the Other. The cross-price elasticity of tobacco in response to alcohol price was found to be greater than the cross-price elasticity of alcohol consumption in the response to change in the price of tobacco (Pierani \& Tiezzi, 2009).

Furthermore, Msambichaka et al. (2018) found that in semi-urban Tanzania there is a correlation between inadequate fruits and vegetables (FV) intake with factors such as young age, being male, low education, low-income occupations, low alcohol, high tobacco, and low healthcare use. Participants who were frequently alcohol consumers were found to be at less risk for daily fruit, vegetable and inadequate FV consumption. The results were the same even after controlling for all socio-demographic variables and this was found to be similar for men and women. On the other hand, this study did not find enough evidence to draw conclusions on FV consumption and smoking.

Previous study also found the association between smoking and excessive alcohol consumption patterns among the rural dwellers, urban dwellers, and rural-to-urban migrants (Taype-Rondan et al., 2017). Taype-Rondan et al., (2017) found that the prevalence of smoking in lifetime was higher in urban residents, intermediary in rural urban migrants, and lower in rural residents. This result indicates that people who are in the process of migrating from rural to urban areas are more likely to engage in smoking behaviours than rural residents. This study argued that migrants are mostly likely to engage in the behaviour of smoking in the process of internal migration.

In relationship perspective, homosexual women are at increased risk for higher consumptions of both tobacco and higher rates of alcohol use compared to heterosexual women (Burgard et al., 2005). According to Burgard et al. (2005), they found that 
women who are classified as lesbian were more likely to report being a current or former tobacco smoker, as compared to heterosexual women who their prevalence was low. One of the reasons behind is the possibility of social stigmatization of homosexuality that is likely to generates stress which contribute to higher rates of tobacco/alcohol use.

Despite many governments' efforts, which are taken by developed countries to control the use of tobacco and alcohol, the problem still persists. Leatherdale et al., (2008) reported the consumption of alcohol, tobacco, and marijuana in Canada to be high among the youths. Basically, there are laws and regulations which prohibit the use of these products at low age, however, they seem not to be effective in controlling consumption in youths' groups. Moreover, this study suggested that alcohol use may proportionally rise the same as tobacco and marijuana use at the young age groups. With the high increasing rate of dangerous substance use, if no further measures are taken, this change in youth behaviour might bring several complications on the control of tobacco, drugs, and its related problem. This study suggests the high probability of youth to consume tobacco or marijuana if they have already consumed alcohol.

Gender has also been one of the most important factors in explaining the alcohol or tobacco consumption pattern. One study found alcohol drinking to have a link with smoking behaviour among men and non-smoking tobacco consumption among women (Banta et al., 2013). The higher prevalence of alcohol consumption was found in men, during ages of 18-25 years. In the beginning of this period the prevalence of smoking was also found to increase by 2- to 3-folds. On the other hand, the less prevalence of consuming alcohol was found in women. Banta et al. (2013) argued that the occurrence of these results tends to be related with the behaviour of chewing betel nuts during the middle age. Other factors which found to have significance in influencing alcohol/tobacco consumption patterns are marriage and marital status of an individual, ethnicity or cultural background, and religion beliefs. These factors raise the possibility of cultural practice, family influence, and contextual influences of accepting alcohol consumption as normal practice during youth or adulthood. To reduce this problem, preventive measures on health and the efforts to provide education as measures to control excessive consumption should then directly target the adolescents and youth.

Furthermore, another study revealed the alcohol consumption behaviour in Tanzania to be higher in Kilimanjaro than in the Mwanza region (Francis et al., 2015). Moreover, this study argues that the reason for higher alcohol consumption in these regions might be influenced by local traditional and cultural practice, especially in the Kilimanjaro region that might have been promoting the consumption of alcohol for social interactions. Generally, there are other studies also which showed cultural influence to be significant in influencing alcohol consumption behaviour, especially in the younger generation (Kuntsche et al., 2006).

In general, excessive consumption of alcohol and tobacco have been among major causes of death worldwide. Approximately 6 million fatalities every year worldwide are the result of side effects caused by cigarette smoking and excessive alcohol drinking behaviour (WHO, 2013). Moreover, cigarette smoking has been a secondary cause to the development of cardiovascular diseases, different cancers, and respiratory problems (Ezzati et al., 2005). On the other hand, alcohol consumption has been associated with occurrence of accidents and development of more than 200 diseases, which costs the world approximately $5.1 \%$ of costs of treatments worldwide (Taype-Rondan et al., 2017).

On household expenditure patterns, previous study found that spending on tobacco positively influences spending on recreation activities, 
transport, spending on alcohol and expenditure on communications (Masaud et al., 2020). On the other hand, the study found out tobacco expenditure tends to reduce expenditure on health, food, and housing. These findings are consistent with many other studies which found that the households which consume tobacco tend to allocate less spending on activities that are more likely to increase their productivity and welfare of the households (Efroymson et al., 2001; John et al., 2012). Masaud et al., (2020) also found that tobacco consuming households to have a higher probability of having children with malnutrition compared to nonsmoking households. The argument behind is tobacco consuming households tend to divert budget from food to tobacco.

Other adverse effects of alcohol and tobacco consumption have been found to be higher risk acquiring debts through borrowing or selling of assets to pay medical expenses among alcohol or tobacco consumers. This situation is also associated with higher risks of being distressed during hospitalization. Moreover, the same study reported the existence of a positive relationship between tobacco and alcohol consumption with the risk of becoming poor because of debts resulting from medical expenses and distress resulting from asset selling to cover medical expenses (Bonu et al., 2005). This behaviour might be associated with a lower savings rate among alcohol and tobacco consumers, where they spend all their savings in those products and therefore when something like diseases attack the family member, the only option is to sell assets to cover those expenses.

Basically, the externalities resulting from public smoking, tend to exceed the revenue which many governments get through tobacco and cigarettes taxation (Khanijahani, 2020). The costs of treating health problems and their related complications resulting from direct or indirect smoking usually exceeds the income which the governments get through taxation and other charges. This situation can be considered as the indication of market failure in the market system of tobacco. The burden in the economy, which is generated directly or indirectly by smoking and excessive alcohol consumption behaviour, is very high and therefore not rational. Therefore, the market will not be efficient if we decide to leave the supply system (producers' side), the demand of the consumers, and consumption behaviour of tobacco and alcohol to the market forces. Intervention is highly needed to reduce negative effects generated by smoking and excessive alcohol consumption behaviour, which includes high death rate and loss of manpower, which results in reductions of productivity.

To contribute efficiently to reducing the above problems, this study examined the influence of socioeconomic and demographic characteristics on alcohol and tobacco spending in Tanzania. Understanding the determinants of alcohol and tobacco spending will help to identify the vulnerable groups and factors which influence alcohol and tobacco spending. This will help the government and policy makers to prepare relevant policies while targeting specific groups which will be more affected by this problem.

\section{METHODOLOGY}

This study used the cross-sectional data from the Household Budget Survey (HBS) of 2017/18 which is provided by the National Bureau of Statistics (NBS) in Tanzania, and they are freely available for public use. 2017-18 HBS data was collected to obtain information on poverty situations in the country and other related characteristics (MoFPPED, 2018). Moreover, another objective of this survey is to measure the progress made in the process of improving the standard of living of the peoples in Tanzania.

HBS has been conducted since 1969. Under the supervision of the NBS, HBS has been conducted in Tanzania Mainland to collect data on household consumption. Moreover, this survey aims at collecting information on household expenditure 
and the poverty situation at household level. Tanzania is divided in two parts, Tanzania mainland which currently (as per year 2021) comprises 26 regions, and Zanzibar (Tanzania islands) which comprises 5 regions.

To analyse the determinants of household spending on alcohol and tobacco, this study used dependent variable 'spending on alcohol and tobacco' which is household monthly expenditure on alcohol and tobacco. The dependent variable is a continuous variable. This study used 4 variables of interests which are 'non-alcohol and tobacco expenditures' as a proxy for income, which is a continuous variable. Variable non-alcohol and tobacco expenditure was created from total expenditure minus household spending on alcohol and tobacco to remove spending on alcohol and tobacco to appear on both right and left side of the equation. Moreover, this study used variable location as a proxy for culture. The assumption is rural areas tend to preserve culture more than urban areas because of low interaction of people, ceteris paribus. Location variable is dummy with the value of 1 if the household lives in rural areas, and 0 otherwise.

Furthermore, this study used variable 'age of household head' as a proxy for measuring change in preference of these addictive products over time. The assumption is (everything remains constant) young people tend to consume more addictive products during young age, but as time going on, because of factors such as increasing awareness of dangers of alcohol and tobacco and changes in their body forbearance on handling the adverse effects of these products due to old age, or health complications, they tend to change their behaviour and reduce excessive consumption of these addictive products. To measure the possible nonlinear changes over time, variable age squared was added. Lastly, variable household size was used as a proxy for level of responsibility with the assumption that the household with large size, tend to have more responsibilities that the household with low size, ceteris paribus.

To analyse the determinants of household spending on alcohol and tobacco, this study used Tobit Regression Model using equation 1.

$\boldsymbol{Y}_{i}^{*}=\boldsymbol{X}_{i}^{T} \boldsymbol{\beta}+$

Where; $\mathrm{Y}^{*}$ is the dependent variable spending on Alcohol and tobacco; $\beta=$ is the coefficient of $\mathrm{Xi} ; \mathrm{X}_{1}$ $=$ non-alcohol and tobacco expenditure (Total household expenditure minus expenditure of alcohol and tobacco as a proxy for income); $\mathrm{X}_{2}=$ Location (as proxy for culture); $\mathrm{X}_{3}$ and $\mathrm{X}_{4}=$ Age of household age and age squared (as a proxy for preference change overtime); $\mathrm{X}_{5}=$ Household size (as a proxy for level of responsibility.

\section{RESULTS}

\section{Summary on Household Spending Structure}

Table 1 shows the mean monthly household expenditure for 15 expenditure categories and their percentage to total expenditure. The highest expenditure category being food consumption with $44.36 \%$ and average budget allocated for alcohol and tobacco in all households being $0.47 \%$ of total household expenditure. 
East African Journal of Business and Economics, Volume 3, Issue 1, 2021

Article DOI: https://doi.org/10.37284/eajbe.3.1.419

Table 1: Household expenditure category and its percentage to total expenditure

\begin{tabular}{lll}
\hline Expenditure $\mathbf{( 9 , 4 6 5}$ observations) & $\begin{array}{l}\text { Mean household } \\
\text { expenditure (Tsh.) }\end{array}$ & $\begin{array}{l}\text { \% on total } \\
\text { expenditure }\end{array}$ \\
\hline Alcohol and tobacco & 1990.144 & 0.47 \\
Clothing and footwear & 18930.19 & 4.47 \\
Fuel (Housing, water, electricity, gas) & 52802.1 & 12.46 \\
Furniture, house equipment and maintenance & 9409.969 & 2.22 \\
Health & 7969.015 & 1.88 \\
Transport & 27313.06 & 6.45 \\
Communication & 10344.48 & 2.44 \\
Recreation and culture & 12648.27 & 2.99 \\
Education & 9380.32 & 2.21 \\
Restaurants and hotels & 1682.532 & 0.40 \\
Miscellaneous goods and services & 15542.02 & 3.67 \\
Durable & 6014.977 & 1.42 \\
Other non-food expenditures & 32871.3 & 7.76 \\
Food consumption & 187964.3 & 44.36 \\
Investment & 11483.33 & 2.71 \\
Total household expenditures & 423713.9 & 100 \\
\hline
\end{tabular}

Table 2 shows the comparison on mean/average household spending between household with spending greater than zero (0) on alcohol and tobacco and households with zero spending. On average, household with greater than zero spending on alcohol and tobacco spend higher percentage of their income in furniture, house equipment's and home maintenance, recreation and culture, education, miscellaneous goods and services, and durable goods compared to those with 0 zero spending on alcohol and tobacco 
Table 2: Comparison of mean expenditure between household with 0 spending on alcohol and tobacco and household with spending greater than 1 on alcohol and tobacco

\begin{tabular}{|c|c|c|c|c|c|}
\hline $\begin{array}{l}\text { Expenditure } \\
\text { 9,465 observations }\end{array}$ & $\begin{array}{l}\text { Mean household } \\
\text { expenditure (TSh) }\end{array}$ & $\begin{array}{l}\% \text { on total } \\
\text { expenditure }\end{array}$ & $\begin{array}{l}\text { Mean household } \\
\text { expenditure (Tsh) }\end{array}$ & $\begin{array}{l}\% \text { on total } \\
\text { expenditure }\end{array}$ & Difference (\%) \\
\hline & \multicolumn{2}{|c|}{0 spending on Alcohol and tobacco } & \multicolumn{3}{|c|}{ >0 Spending on Alcohol and tobacco } \\
\hline Variable & Mean & $\%$ & & & \\
\hline Alcohol and tobacco & 0 & 0.00 & 14808.74 & 3.03 & $(3.03)$ \\
\hline Clothing and footwear & 18080.45 & 4.37 & 24403.4 & 5.00 & $(0.63)$ \\
\hline $\begin{array}{l}\text { Fuel (Housing, water, electricity, } \\
\text { gas) }\end{array}$ & 52548.59 & 12.70 & 54434.96 & 11.15 & 1.55 \\
\hline $\begin{array}{l}\text { Furniture, house equipment and } \\
\text { maintenance }\end{array}$ & 9006.298 & 2.18 & 12010.03 & 2.46 & $(0.28)$ \\
\hline Health & 7980.122 & 1.93 & 7897.479 & 1.62 & 0.31 \\
\hline Transport & 27161.88 & 6.57 & 28286.82 & 5.80 & 0.77 \\
\hline Communication & 10280.79 & 2.48 & 10754.68 & 2.20 & 0.28 \\
\hline Recreation and culture & 8609.046 & 2.08 & 38665.08 & 7.92 & $(5.84)$ \\
\hline Education & 9074.299 & 2.19 & 11351.42 & 2.33 & $(0.13)$ \\
\hline Restaurants and hotels & 1681.294 & 0.41 & 1690.507 & 0.35 & 0.06 \\
\hline Miscellaneous goods and services & 15081.26 & 3.65 & 18509.84 & 3.79 & $(0.15)$ \\
\hline Durable & 5521.813 & 1.33 & 9191.465 & 1.88 & $(0.55)$ \\
\hline Other non-food expenditures & 35145.26 & 8.49 & 18224.65 & 3.73 & 4.76 \\
\hline Food consumption & 184286.6 & 44.54 & 211652 & 43.37 & 1.17 \\
\hline Investment & 11257.01 & 2.72 & 12941 & 2.65 & 0.07 \\
\hline Total household expenditures & 413732.4 & 100 & 488005.2 & 100 & $(18)$ \\
\hline
\end{tabular}


Table 3 shows the summary statistics of variables used to analyse the determinants of household spending on alcohol and tobacco in Tanzania.

Table 3: Summary of variables

\begin{tabular}{lrrlll}
\hline Variable & Obs & Mean & $\begin{array}{l}\text { Std. } \\
\text { Dev. }\end{array}$ & Min & Max \\
\hline$Y^{*}$ (Spending on Alcohol and tobacco in Tsh) & 9,465 & 1990.144 & 10360.89 & 0 & 304166.7 \\
\hline $\begin{array}{l}X_{1} \text { (Non-alcohol and tobacco expenditures, in } \\
\text { TZS) }\end{array}$ & 9,465 & 421723.8 & 1693137 & 5434.524 & $1.50 \mathrm{e}+08$ \\
\hline$X_{2}$ (Location (Urban/Rural)) & 9,465 & .7053354 & .4559159 & 0 & 1 \\
\hline$X_{3}$ (Age of HH head) & 9,465 & 47.06223 & 15.74134 & 13 & 112 \\
\hline$X_{4}$ (Age squared) & 9,465 & 2462.617 & 1667.065 & 169 & 12544 \\
\hline$X_{5}$ (Household size) & 9,465 & 4.853143 & 2.910831 & 1 & 38 \\
\hline
\end{tabular}

Table 4 present the Tobit regression results for the determinants of alcohol and tobacco spending in Tanzania. The dependent variable is spending on alcohol and tobacco, the independent variables are non-alcohol and tobacco expenditure as a proxy for household income, location (rural/urban) as a proxy for culture, age of household head and age squared as a proxy to measure changes in preference to alcohol and tobacco over time, and household size as a proxy of poverty and level of responsibility. All independent variables except household size are significance to explain the correlation on dependent variable.

Table 4: Tobit regression results for determinants of alcohol and tobacco spending

\begin{tabular}{ll}
\hline Dependent variable: Spending on Alcohol and tobacco & Tobit $(\mathbf{b} / \mathbf{s e})$ \\
\hline X1 (Non-alcohol and tobacco expenditures) & $0.001 *(0.00)$ \\
X2 (Location (Urban/Rural)) & $10793.714 * * *(1486.22)$ \\
X3 (Age of HH head) & $726.028 * * *(218.71)$ \\
X4 (Age squared) & $-5.871 * *(2.04)$ \\
X5 (Household size) & $338.147(204.94)$ \\
constant & $-73642.467 * * *(5728.90)$ \\
$\operatorname{var}(\mathrm{e} . \mathrm{nf} 2)$ & $1.466 \mathrm{e}+09 * * *(66787854.67)$ \\
\hline$* p<0.05, * * p<0.01, * * * p<0.001$ &
\end{tabular}

\section{DISCUSSION}

Income has been one of the important elements in determining the spending behaviour of different expenditure categories in the households. As the findings shows, income is one of the significant factors in explaining spending on alcohol and tobacco in Tanzania. The results show a positive correlation between alcohol and tobacco spending, and household income. That means expenditure on alcohol and tobacco are more likely to increase as household income increases.

Basically, ceteris paribus an increase of income tends to increase the affordability of different goods and services in the household, including alcohol and tobacco. This can also be the case for Tanzania where the government has continued to cut excise tax on alcohol and cigarettes in the previous years, and even in the national budget of financial year 
2021/22, the government of the United Republic of Tanzania has reduced the excise tax for local malted beers which uses domestic raw materials from 765 TZS to TZS 620 per litre (KPMG, 2021). The decision to cut the excise tax for approximately $18 \%$ will eventually continue to lower the price of alcohol and increase the affordability of consumer to buy alcohol and tobacco. Previous literature have revealed that lower price of alcohol tend to increase consumption as consumers will be able to buy more amount of alcohol than before when the price was higher (Cook et al., 2011).

Alcohol and tobacco consumption are among the most important elements in African culture. This being the case for Tanzania as an African country, alcohol and tobacco have been part of many Tanzania tribes for the long time. Alcohol have been used in different cultural occasions including celebration and mourning activities. Moreover, majority of Tanzania tribes who are agriculturalists, have been using alcohol for social gathering approximately every day after farming and discuss about their day and exchanging ideas. Majority of people in Tanzania lives in rural areas, where in different parts of the world, rural areas have been known for preserving the culture than urban areas. Basically, high consumption of alcohol and tobacco in rural areas can also be influenced by social and cultural acceptability of these addictive products as the part of their daily life (Dixon \& Chartier, 2016).

As supported by the results, culture is one of the significant factors on explaining spending behaviour on alcohol and tobacco in Tanzania. The result indicates a positive correlation between living in rural areas and expenditure on alcohol and tobacco. That means households in rural areas are more likely to spend on alcohol and tobacco compared to the households living in urban areas. These finding are also supported by the study by Francis et al., (2015) who argued that high consumption of alcohol in Kilimanjaro and Mwanza regions of Tanzania might be influenced by local traditional and cultural practices. As urban areas have been argued to lose culture overtime because of modernity and interactions of peoples from different cultures, people in urban areas also seems to lose the culture of consuming alcohol and tobacco by having a less likelihood of spending on those products compared to peoples in rural areas as indicated by the results.

Consumption of tobacco or alcohol have not been the same over time at all age groups. This being the case, we can say that the relationship between time and these addictive substances is not linear. As the results shows, spending on alcohol and tobacco in Tanzania tend to increase as age increase. However, over time as age increase, spending tends to decrease again. These changes might be associated with change in preference on these products, awareness, and health concern whereas people becoming older, they tend to decrease consumption of these products because their bodies cannot tolerate more the side effects resulted from using alcohol and tobacco. Existence of this situation can also be supported by the study of Banta et al., (2013) who found the increasing behaviour of smoking is not linear as differ over time, after finding the prevalence of smoking to be 2-3 times higher at the age of 18-25 compared to other age categories. Moreover, Amalia et al., (2019) also found the prevalence of smoking in a lifetime to be not linear as it was higher at the age of 51 and below compared to the age above 51 .

This study did not find enough evidence to comment on the influence of level of household responsibility on spending on alcohol and tobacco because variable household size which was used as proxy to measure level of responsibilities was not significant.

\section{CONCLUSION AND RECOMMENDATION}

\section{Conclusion}

Alcohol and tobacco are among the important products in the economy. This is because these products provide income to the governments through taxation and different charges. Moreover, 
tobacco provides raw materials for cigarettes, cigar industries and other related products. Furthermore, alcohol production industries provide markets for agricultural products like cereals and fruits which are used as raw materials for production of many alcohol beverages. These products together provide employment opportunities for many workers from raw material production up to industries during processing. In addition, these products generate profits to many businessmen from industrial owners to retailers who sell them to final consumers.

However, when these products are excessively consumed, they cause dangerous effects from individuals to national level in general. This is because of its influences on diseases (Ezzati et al., 2005), accidents (Taype-Rondan et al., 2017), and loss of manpower, which decrease productivity and increase dependency resulting from accidents and diseases. These problems call for necessary measures to mitigate or reduce these effects from happening. To do that, this study analysed household spending behaviour on these addictive products in Tanzania to prepare a base to mitigate the effects of alcohol and tobacco.

This study found income, cultural influence, and changes of preference overtime to be significant factors that influence household spending on alcohol and tobacco overtime. That means, people with high income are more likely to spend on alcohol and tobacco than people with low income. Probably people with high income tend to have more disposable income than people with low income that allows them to spend more on alcohol and tobacco than people with low income. Moreover, culturally people in rural areas are more likely to preserve the culture of using alcohol in social gathering and different celebrations than people in urban areas. In addition, the preference of using these products overtime might change as age increase because of different factors like awareness and health complication. This study did not find enough evidence to conclude on the influence of responsibility on spending on alcohol and tobacco.
The result of this study shows only correlation and not causal influence between the dependent and independent variables.

\section{Policy Recommendation}

Because of limitation of data, this study could not conclude on factors that can influence the supply of these products. Therefore, the recommendation of this study is basing only on the demand side. Cultural activities of Tanzania are highly associated with alcohol or tobacco consumption. Whenever there is social event, alcohol is among important product to make peoples enjoy their time together. Even for youth, consuming alcohol or smoking is among things which make them feels adult. This might be associated with limited availability of activities which can make people enjoy their time without using these addictive products. Therefore, to ensure long term results in managing spending and consumption of these products, there is a need to use measures which can help peoples to diverge their demand from these products and change to activities which does not involve the use of alcohol or tobacco.

Due to the influence of culture in alcohol and tobacco spending, the government need to increase campaign on the risks of alcohol and tobacco consumption especially in rural areas to help increasing the awareness about the dangers of excessively consuming these addictive products. Previous literature revealed that peoples who have awareness about the dangers of tobacco on their health, tend to have less likelihood of consuming this addictive products (John et al., 2012). In addition, previous literature found alcohol and tobacco to be complementary goods and therefore, any measure to reduce one of the two products will have impact on the other product (Pierani \& Tiezzi, 2009). Moreover, advising peoples to engage in other activities like sport and games might help to change the culture and behaviour of consuming alcohol or tobacco during free time. Previous literature revealed that engaging in physical and 
club activities can have positive effects in influencing drug-free lifestyle (Leaver-Dunn et al., 2007).

Affordability due to increasing income resulted from tax reduction need also to be controlled. Instead of decreasing excise tax, the government can increase. Previous literature revealed the inverse relationship between higher price and expenditure on alcohol (Elder et al., 2010). However, further research needs to be conducted to determine the magnitude on how tax rate will affect spending in Tanzania context. In addition, despite of having big corporations and industries producing alcohol and tobacco in low- and medium-income countries, traditional and local homemade alcohol has been among the suppliers of alcohol in low and medium income countries (Walls et al., 2020). This is also the case for Tanzania, especially in rural areas where the accessibility of local and traditional home-made alcohol is very high, and therefore need to be controlled.

To strengthening this contribution on reduction of household spending alcohol and tobacco, further studies need to be conducted to analyse the relationship between price of these products, spending and consumptions. This is because of the difference between spending and consumption. There are some people who spend more on these products more than they consume. Also, the existence of price differences between the urban and rural areas and difference of product availability in those areas, need further research to strengthen this contribution.

\section{REFERENCES}

Ahmed, A. T., Karter, A. J., \& Liu, J. (2006). Alcohol consumption is inversely associated with adherence to diabetes self-care behaviours. Diabetic medicine, 23(7), 795-802

Alkan, Ö., \& Abar, H. (2020). Determination of factors influencing tobacco consumption in Turkey using categorical data analyses1. Archives of environmental \& occupational health, 75(1), 27-35.

Amalia, B., Cadogan, S. L., Prabandari, Y. S., \& Filippidis, F. T. (2019). Socio-demographic inequalities in cigarette smoking in Indonesia, 2007 to 2014. Preventive medicine, 123, 27-33.

Banta, J. E., Addison, A., Job, J. S., Yel, D., Kheam, T., \& Singh, P. N. (2013). Patterns of alcohol and tobacco use in Cambodia. Asia Pacific Journal of Public Health, 25(5_suppl), 33S-44S.

Bonu, S., Rani, M., Peters, D. H., Jha, P., \& Nguyen, S. N. (2005). Does use of tobacco or alcohol contribute to impoverishment from hospitalization costs in India?. Health Policy and Planning, 20(1), 41-49.

Burgard, S. A., Cochran, S. D., \& Mays, V. M. (2005). Alcohol and tobacco use patterns among heterosexually and homosexually experienced California women. Drug and alcohol dependence, 77(1), 61-70.

Cook, P. A., Phillips-Howard, P. A., Morleo, M., Harkins, C., Briant, L., \& Bellis, M. A. (2011). The Big Drink Debate: perceptions of the impact of price on alcohol consumption from a large scale cross-sectional convenience survey in north west England. BMC Public Health, 11(1), $1-11$.

Dixon, M. A., \& Chartier, K. G. (2016). Alcohol use patterns among urban and rural residents: demographic and social influences. Alcohol research: current reviews, 38(1), 69

Efroymson, D., Ahmed, S., Townsend, J., Alam, S. M., Dey, A. R., Saha, R., ... \& Rahman, O. (2001). Hungry for tobacco: an analysis of the economic impact of tobacco consumption on the poor in Bangladesh. Tobacco control, 10(3), 212-217

Elder, R. W., Lawrence, B., Ferguson, A., Naimi, T. S., Brewer, R. D., Chattopadhyay, S. K., ... \& 
Task Force on Community Preventive Services. (2010). The effectiveness of tax policy interventions for reducing excessive alcohol consumption and related harms. American journal of preventive medicine, 38(2), 217-229.

Ezzati, M., Henley, S. J., Thun, M. J., \& Lopez, A. D. (2005). Role of smoking in global and regional cardiovascular mortality. Circulation, 112(4), 489-497

Francis, J. M., Weiss, H. A., Mshana, G., Baisley, K., Grosskurth, H., \& Kapiga, S. H. (2015). The epidemiology of alcohol use and alcohol use disorders among young people in northern Tanzania. PloS one, 10(10), e0140041

John, R. M. (2005). Tobacco consumption patterns and its health implications in India. Health policy, 71(2), 213-222

John, R. M. (2006). Household's tobacco consumption decisions: Evidence from India. Journal of South Asian Development, 1(1), 101-126

John, R. M., Ross, H., \& Blecher, E. (2012). Tobacco expenditure and its implications for household resource allocation in Cambodia. Tobacco control, 21(3), 341-346.

Khanijahani, A. (2020). Governance and public health: implications for targeting smoking in the United States. International Journal of Health Governance, 25(2), 151-159. https://doi.org/10.1108/IJHG-06-2019-0041

KPMG. (2021). 2021-22 Tanzania Budget Brief. Https://Home.Kpmg, June 2021, 1-13. https://assets.kpmg/content/dam/kpmg/ke/pdf/ta x/2021/Budget-brief-2021-22-Tanzaniafinal.pdf

Kuntsche, E., Knibbe, R., Gmel, G., \& Engels, R. (2006). Who drinks and why? A review of sociodemographic, personality, and contextual issues behind the drinking motives in young people. Addictive behaviors, 31(10), 1844-1857

Leatherdale, S. T., Hammond, D., \& Ahmed, R. (2008). Alcohol, marijuana, and tobacco use patterns among youth in Canada. Cancer Causes \& Control, 19(4), 361-369

Leaver-Dunn, D., Turner, L., \& Newman, B. M. (2007). Influence of sports' programs and club activities on alcohol use intentions and behaviors among adolescent males. Journal of Alcohol and Drug Education, 51(3), 57.

Masa-Ud, A. G. A., Chelwa, G., \& van Walbeek, C. (2020). Does tobacco expenditure influence household spending patterns in Ghana?: Evidence from the Ghana 2012/2013 Living Standards Survey. Tobacco induced diseases, 18.

MoFP-PED. (2018). Ministry of Finance and Planning - Poverty Eradication Division (MoFP- PED) [Tanzania Mainland] and National Bureau of Statistics (NBS), 2019. Tanzania Mainland Household Budget Survey 2017-18, Key Indicators Report. Dodoma, Tanzania. (Vol. 1, Issue 1). https://www.nbs.go.tz/index.php/en/censussurveys/poverty-indicators-statistics/householdbudget-survey-hbs/477-the-2017-18-householdbudget-survey-dataset

Msambichaka, B., Eze, I. C., Abdul, R., Abdulla, S., Klatser, P., Tanner, M., ... \& Probst-Hensch, N. (2018). Insufficient fruit and vegetable intake in a low-and middle-income setting: a populationbased survey in semi-urban Tanzania. Nutrients, 10(2), 222.

Pierani, P., \& Tiezzi, S. (2009). Addiction and interaction between alcohol and tobacco consumption. Empirical Economics, 37(1), 1-23

Swahn, M. H., Bossarte, R. M., West, B., \& Topalli, V. (2010). Alcohol and drug use among gang 
members: Experiences of adolescents who attend school. Journal of School Health, 80(7), 353-360

Taype-Rondan, A., Bernabe-Ortiz, A., Alvarado, G. F., Gilman, R. H., Smeeth, L., \& Miranda, J. J. (2017). Smoking and heavy drinking patterns in rural, urban and rural-to-urban migrants: the PERU MIGRANT Study. BMC public health, 17(1), 1-10.

Walls, H., Cook, S., Matzopoulos, R., \& London, L. (2020). Advancing alcohol research in lowincome and middle-income countries: a global alcohol environment framework. BMJ global health, 5(4), e001958.

World Bank. (2019). Tobacco. https://www.worldbank.org/en/topic/tobacco\#1, Accessed on 20/01/2021

World Health Organization (WHO). (2014). Global status report on alcohol and health, 2014: World Health Organization. Geneva, Switzerland..

World Health Organization (WHO). (2013). WHO report on the global tobacco epidemic, 2013: enforcing bans on tobacco advertising, promotion and sponsorship. World Health Organization. 